\title{
QUASAR RADIO STRUCTURE IN CLUSTER ENVIRONMENTS
}

\author{
J.B.HUTCHINGS \\ Dominion Astrophysical Observatory \\ Victoria, B.C., Canada \\ AND \\ A.C.GOWER, S.RYNEVELD AND A.DEWEY \\ Dept of Physics and Astronomy \\ University of Victoria, B.C., Canada
}

We have VLA snapshots at $6 \mathrm{~cm}$ and $20 \mathrm{~cm}$ of $\sim 50$ QSOs of redshift $\leq 0.7$ with a range of known optical galaxy environments. The radio sources were characterised by measures of flux, size, and shape. The cluster density of the QSOs is given by the $\mathrm{B}_{g q}$ number from galaxy companion data largely published by Yee and Ellingson (e.g. ApJ 411, 43, 1993).

Number of sources. In several of the fields, one or two other sources were detected. We limit our discussion of these sources to within 200 arcsec of the map centre (the QSO) to avoid (50 $\mathrm{MHz}$ ) bandwidth smearing at larger angular distances.

All the extra sources lie at distances beyond the known galaxy companions. Thus, we do not yet know if they are associated or background sources. As a comparison, we count the sources detected in our maps from a separate program of 6 very rich (X-ray selected) clusters. In all cases the central cD galaxy was one of the strongest sources. We thus counted extra sources around the $\mathrm{cD}$ galaxies, with the same limiting flux and area limits as in our QSO clusters.

The numbers aside from the central source are: mean QSO cluster 0.7; typical rich cluster 1.6; exceptional rich cluster (A2390) 5. Thus, the rich clusters appear to contain on average more sources than the QSO clusters. Here too we do not yet know in general if they are cluster members or some background population (enhanced by lensing?). However, in the exceptional cluster A2390, most of the extra sources are not identified with cluster members (Abraham et al Ap.J. 1996 preprint), or any optically visible object . 
The mean $\mathrm{B}_{g q}$ of the rich clusters is 1400 , compared with 300 for the QSO sample, although some of the QSO clusters have $\mathrm{B}_{g q}$ near to 1000 . There is no correlation between $B_{g q}$ and number of sources in the QSO sample.

Cluster environment. The distribution of QSO $\mathrm{B}_{g q}$ values has a roughly Gaussian spread about zero, with $\sigma \sim 350$, and a tail to higher values. Values below 350 are consistent with zero, and the remainder are significantly clustered. In order to use a subset matched in redshift for high and low $\mathrm{B}_{g q}$, we use QSOs with redshift $>0.4$. The table shows values of properties in this matched sample of 37 .

\begin{tabular}{llll}
\hline Property & $\begin{array}{l}\mathrm{B}_{g q}>350 \\
\text { mean (\& median) }\end{array}$ & $\mathrm{B}_{g q}<350$ & $\begin{array}{l}\text { Difference } \\
\left.\text { (high } \mathrm{B}_{g q}-\text { low } \mathrm{B}_{g q}\right)\end{array}$ \\
\hline $\mathrm{B}_{g q}$ & $833(792)$ & $37(0)$ & (defining cut) \\
$\mathrm{z}$ & $0.55(0.58)$ & $0.52(0.51)$ & - \\
$\mathrm{m}_{v}$ & $17.6(17.0)$ & $17.6(17.2)$ & - \\
Lobe dominance (1-4) & $3.5(4)$ & $2.7(3)$ & $\sim 1$ \\
Size (kpc) & $162(134)$ & $142(135)$ & resolved sources: - \\
& & 100 & all sources: larger \\
Bend angle $\left(^{\circ}\right)$ & $8(6)$ & $16(9)$ & less bent? \\
Lobe length ratio & $0.72(0.72)$ & $0.71(0.65)$ & - \\
Source complexity (1-5) & $2.8(3)$ & $3.0(3)$ & - \\
$\alpha_{\text {core }}$ & $-0.08(-0.12)$ & $-0.12(-0.03)$ & - \\
log P (W/Hz) mean core & $25.4(25.3)$ & $25.6(25.5)$ & $-0.2(-0.2)$ \\
$\quad$ mean lobe & $25.6(25.7)$ & $25.2(25.2)$ & $0.4(0.5)$ \\
\hline
\end{tabular}

The data suggest that the high $\mathrm{B}_{g q}$ group has:

1. More lobe-dominated type (all compact sources have low $\mathrm{B}_{g q}$ )

2. Higher lobe luminosities (by a factor 2-3)

3. Lower core luminosities (by a factor $<2$ ) and possibly

4. Larger source size (if unresolved sources are included)

5. Less bent sources (mainly because two very bent ones are low $\mathrm{B}_{g q}$ )

K-S tests indicate that $1-5$ above are significant at the $2 \sigma$ to $3 \sigma$ level. There are also significant continuous correlations with $\mathrm{B}_{g q}$ for the powers. The lobe length ratio, source complexity, core spectral index, and number of additional sources show no dependence on $\mathrm{B}_{g q}$.

These results will be published in full elsewhere. 